\title{
Recommender System in Tourism Using Case based Reasoning Approach
}

\author{
Tamir Anteneh Alemu \\ Faculty of Computing, Bahir Dar Institute of Technology, Bahir Dar, Ethiopia \\ Email: tamirat.1216@gmail.com \\ Alemu Kumilachew Tegegne \\ Faculty of Computing, Bahir Dar Institute of Technology, Bahir Dar, Ethiopia \\ Email: alemupilatose@gmail.com \\ Adane Nega Tarekegn \\ Faculty of Computing, Bahir Dar Institute of Technology, Bahir Dar, Ethiopia \\ Email: nega2002@gmail.com
}

Received: 07 April 2017; Accepted: 13 May 2017; Published: 08 September 2017

\begin{abstract}
Using recommender systems with the help of computer systems technology to support the Tourist advising process offers many advantages over the traditional system. A knowledge based recommender reasons about the fit between a user's need and the features of available products. Providing an effective service in Ethiopian Tourism sector is critical to attract more foreign and local tourists. However, there are major problems that need immediate solution. First, the difficulty of getting fast, reliable, and consistent expert advice in the sector that is suitable to each visitor's characteristics and capabilities. Second, inadequacy of the number of experienced experts and consulting individuals who can give advice on tourism issues in the country. Therefore, this paper aims to design a recommender system for tourist attraction area and visiting time selection that can assist experts and tourists to make timely decisions that helps them to get fast and consistent advisory service. So that, visitors can identify tourist attraction areas that have the highest potential of success/satisfaction and that match their personal characteristics. The system provides recommendation to visitors based on previously solved cases and new query given by the tourist. For this study, about 615 cases which are collected from National Tour operation and 10 attributes which are collected from experts are used as case base. These attributes and cases are used as knowledge base to construct case based recommender. The system calculates similarity between existing cases and new queries that are provided by the visitors, and provide solution or recommendation by taking best cases to the new query. In this study, JCOLIBRI case base development tool is used to develop the prototype. JCOLIBRI contains both user interface which enables visitors to enter their query and programming codes with the help of Java script language. To decide the applicability of the prototype in the domain area, the system has been evaluated by involving domain experts and visitors through visual interaction using the criteria of easiness to use, time efficiency, applicability in the
\end{abstract}

domain area and providing correct recommendation. Based on prototype user acceptance testing, the average performance of the system is $80 \%$ and $82 \%$ by domain experts and visitors respectively. The performance of the system is also measured using the standard measure of relevance (IR system) recall, precision and accuracy measures, where the system registers $83 \%$ recall, $61 \%$ precision and $85.4 \%$ accuracy.

Index Terms-Recommender system, case based reasoning $(\mathrm{CBR})$, tourism, Ethiopia

\section{INTRODUCTION}

We are often making choices without sufficient personal experience of the alternatives that are available to us in different circumstance. Nowadays it is very important for people to be supported in their decisions, due to the exponential increase of the available information. This exponential growth of information creates information overload. However, the term is defined, there cannot be many people who have not experienced the feeling of having too much information which uses up too much of their time, causing them to feel stressed which, in turn, affects their decision-making. i.e people may tend to be reluctant in making decision or they may leads to wrong decision [1].

Recommender systems have proven to be an important response to such a problem by providing users with more proactive and personalized information services. It usually track user's behavior and collect information about items that the user seems to be interested in. So that, they can build a model of what user likes [19]. Recommender systems attempt to reduce information overload and retain customers by selecting a subset of items from a universal set based on user preferences. Case based recommender system is a part of knowledge based recommender system that exploits case based reasoning to generate personalized recommendations for 
exploiting the knowledge contained in past recommendation cases [2].

Tourism is one of the largest and rapidly growing industries in the world, and is even considered by the UN World tourism Organization as the biggest industry in the world when related and complementary industries are taken into consideration. Ethiopia has immense tourism potential owing to its natural, historical and cultural endowments and the flow of tourists in the country becomes increasing from time to time [6]. But there are a number of problems in Ethiopian Tourism faced by Experts in the sector and tourists alike. the problems faced by experts in the sector are lack of appropriate, relevant and understandable information that they need to give advice and guidance to their clients.

As stated by [3], advice is one of the most important problems of the tourism sector because the sector also uses traditional advisory system of tourists. Tourists tend to lose money by making the wrong choices of areas to visit and the wrong time based on their characteristics or location of the area or income level of the tourist. The main problem in Ethiopian tourism sector is, the advising services given are not fast. Because of this, it takes long time to get the required tourist attraction areas and permissions to visit. Difficulty of getting appropriate advice is a critical issue for tourists since knowing the right tourist attraction area is a key factor and also knowing the right time to visit in is another factor to consider new tourists [7]. Therefore, this study aims to design a recommender system that helps visitors to get fast and consistent advisory service so that, visitors can identify tourist attraction areas that have the highest potential of success/satisfaction and that match their personal characteristics.

\section{The COMPARISON OF CASE BASED AND Rule BASED REASONING}

\section{A. Case Based Reasoning}

A case typically represents the description of a problem situation together with the experiences gathered during the solution of the problem situation. It may also contain other items such as the effects of the solution applied or a justification for the solution and explanation or be enriched by an administrative part (including e.g., a case number) [13]. A case-based reasoning (CBR) system is a problem solver that uses the recall of examples as the fundamental problem-solving process. It also contains a number of different knowledge containers like the case base, the vocabulary in which cases are described, the similarity measure used to compare cases, and, if necessary, the knowledge needed to transform recalled solutions. Case-based reasoning relates to a reasoning process based on recalling a related previous experience (a memory of stored cases recording specific prior episodes) rather than reasoning based on generalized rules It can also means using old experiences to understand and solve new problems. In case-based reasoning, a reasoner remembers a previous situation similar to the current one and uses that to solve the new problem [4].

Conceptually case based reasoning (CBR) is commonly described by the CBR-cycle. This cycle comprises four activities [8].

RETRIEVE the most similar case or cases.

REUSE the information and knowledge in that case to solve the problem.

REVISE the proposed solution.

RETAIN the parts of this experience likely to be useful for future problem solving.

In CBR, nearest-neighbor retrieval technique is used to measure similarity between the source case and the case which we are searching. The similarity of the problem (target) case to a case in the case-library for each case attribute is determined. This measure may be multiplied by a weighting factor. Then the sum of the similarity of all attributes is calculated to provide a measure of the similarity of the case in the case-base to that of the target case [9].

Similarity $(\mathrm{T}, \mathrm{S})=\sum \mathrm{f}(\mathrm{Ti}, \mathrm{Si}) \mathrm{X} \mathrm{Wi}$,

Where:

$\mathrm{T}$ - Target case

S- Source case

$\mathrm{i}$ - An individual attribute from 1 to $\mathrm{n}$

f- Similarity function for attribute $i$

W- Weight of attribute $\mathrm{i}$

The similarity of the problem (target) case to a case in the case-library for each case attribute is determined. It is possible to use the unification mechanism directly as matching operation to retrieve similar cases [10].

\section{B. Rule based Reasoning}

Rule based reasoning: Symbolic rules are one of the most popular knowledge representation and reasoning methods. Their popularity stems mainly from their naturalness, which facilitates comprehension of the represented knowledge.

The basic form of a rule is the following:

If <conditions>

Then <conclusion>

Where <conditions> represents the conditions of a rule, whereas <conclusion> represents its conclusion. The conditions of a rule are connected between each other with logical connectives such as AND, OR, NOT etc., thus forming a logical function. When sufficient conditions of a rule are satisfied, the conclusion is derived and the rule is said to fire (or trigger). Rules represent general knowledge regarding a domain [11].

Rules are suitable to represent general knowledge, whereas cases are suitable for representing specific situations. Rules in a rule based system have the abilities to represent experiential knowledge acquired from experts in a direct fashion. Cases are capable of representing specific historical knowledge. The problem here is that it is difficult to acquire complete and perfect knowledge in a complex domain. Cases are natural and easy to obtain. They can be collected from the historical record, repair logs or other sources [11]. CBR uses partial matching to draw a conclusion. If some of the given 
problem descriptions match with a given case, then the case is applicable to the proposed solution. It also tries to handle novel problems by referring previously solved cases. Rule based reasoning uses perfect matching to apply a rule for a given problem.

\section{PROBLEM DESCRIPTION}

As stated [3], advice is one of the most important problems of the tourism sector because the sector also uses traditional advisory system of tourists and the traditional nature of the existing advisory system make it interesting to undertake the study. Lack of access to appropriate information is encountered by Ministry of Culture and Tourism due to the fact that information system is not developed to enable proper collection, organization and dissemination in the sector. In addition, there is no integration or collaboration among different experts that are found in different tourism sectors to develop an organized guidance to new tourists. Because collection of ideas from different tourists is important to develop well defined and organized guidelines for visitors. For instance, one expert may have awareness about natural tourist attraction areas but have no more idea about historical tourist attraction areas, etc. These shows experts advice is limited only with the one most familiar with them[12].

In the context of visiting, the wise words of the oracle emphasize that success depends on ensuring that your visiting strategy fits your personal characteristics [7]. Even though all visitors are trying to get satisfaction, each one comes from a diverse background and has different needs and capabilities. It follows that specific visiting vehicles and methods are suitable for certain types of visitors.

According to the interview made with Ministry of Culture and Tourism development promotion expert, tourists have a few factors to consider, when looking for the right place to visit such as age, nationality, gender, travel frequency, attraction preference and current income level. The expert further comments that due to lack of knowledgeable domain experts to give appropriate advice in Ministry of Culture and Tourism, visitors are confused about where to visit, which route they should follow, and which tourist attraction area is best to them to be satisfied/successful in their recreational program. In developing countries, like Ethiopia, the availability of specialists/experts who can provide an effective service in the tourism sector is also a problem in many small towns and villages and recommender systems can prove to be indispensable in such conditions.

\section{RELATED WORKS}

Even though, we are often making choices without sufficient personal experience of the alternatives that are available to us in different circumstances, in our everyday life, we sometimes rely on recommendations from other people either by word of mouth, recommendation letters or on movie and book reviews to select from the huge amount of Information that is available in different places but this suggestion is not enough in this digital age [5].

One of the related works is "An Automated University Admission Recommender System for Secondary School Students" by Fong and [14]. Admission and placement of students is based on the perspective of Universities who knows little about the incoming student background but not based on the perspective of high schools who knows the detail of their students. There is value in extending the university admission process to include secondary schools [14].

In this work, the author proposes a novel design of a recommender system that can provide recommendations about which universities a student should apply to, taking not only the student's secondary school scores but also other factors such as background interest and special skill into account. In the summary of the author, education systems which do not have a standardized open exam for university admissions face the challenges of matching the right secondary school students with the right universities and field of studies and the ways that they should enter. This implies some manual processes are needed and web based recommendation system is very important for decision making. To do that, the author applied a hybrid data mining model to implement a recommender system prototype and analyze different data from secondary schools.

In addition to that, a work entitled "Recommender system for higher education" has been done to discuss the process of developing recommender system for educational institutions. The system is web based application that guides students for decision making based on their personal test. Information about course, curriculum research and facilities in the field of education is important to be available on the web. Clear information about educational activities with description enables students, partners and people to choice more efficiently and scientifically to make right decision [15].The author concludes that, we can make beneficial use of artificial intelligence techniques like database design and selection, content based recommendations, user profiling, integrating groups of users with similar interests and integrating the domain knowledge and expertise. Hybrid recommendation system approach is important in educational institutions [15].

The paper [6], also tried to discuss about course recommendation using data mining techniques called association rule. According to the author, students often need guidance in choosing adequate courses to complete their academic degrees. Course recommender systems have been suggested in the literature as a tool to help students make informed course selections.

Students who join higher education degrees are faced with two main challenges: a myriad of courses or field of studies from which to choose, and a lack of knowledge about which courses or field of studies are relevant to follow and in what sequence. Mostly, it is according to their friends and colleagues' manual recommendations 
that the majorities of them choose their field 40 of study and register for it. It would be useful to help students in finding courses of interest by the intermediary of web based recommender system [16].

The main focus of the authors was on the effectiveness of the incorporation of data mining in course recommendation. The system is based on the following collaborative filtering algorithms: user-based and itembased. According to the author, the system can predict the usefulness of courses to a particular student based on other users' course ratings. To get accurate recommendations, one must evaluate as many courses as possible. Based on the evaluation results, the author suggests $\mathrm{C} 4.5$ as the best algorithm for course recommendation. The system cannot predict recommendations for students who have not taken any courses at the University. Generally, there are many recommender systems developed globally and few attempts have been done locally on the area of recommender system in different sectors.

To conclude, several studies have been developed in recommender systems to the solution of a particular problem. But, as far as the researcher's knowledge, no one has made an attempt to develop systems that can provide possible recommendations on tourist attraction area selection. Moreover, most of the recommender system doesn't provide explanation facility on the specified domain area. Thus, in this study an attempt is made to design a case based recommender system for tourist attraction area selection that can provide recommendation for visitors about the recommended attraction area with the appropriate time period and also give explanation facility about it.

\section{Methodology of THE StUdy}

The following methodologies have been used in the course of this study to achieve the above stated research objective.

\section{A. Data source}

The main data source used for this study was domain Experts working at MoCT (Ministry of Culture and Tourism) and NTO (National Tour Operation) as well as previously solved cases which are available in the aforementioned organizations. The Researcher's selects these organizations since they use traditional advisory system of tourists and the traditional nature of the Existing advisory system makes it interesting to undertake the study.

\section{B. Data collection Methods}

To collect the required domain knowledge, both primary and secondary data collection methods have been employed. As primary sources, Tourism experts from MoCT\& NTO and tourists from different tourism sectors have been interviewed. In addition, relevant literature from all possible sources including journal articles, tourism related websites, manuals especially on Ethiopian tourism, and guidelines has been reviewed. To acquire the required tacit knowledge from the selected domain expert, the researcher has employed semistructured interview technique which focuses on the concept, procedures, and guidelines as well experience which domain expert used while advising in tourists. The researcher's selects semi-structured interview technique because it allows the interviewer to change the order of the questions and add new question based on the participant response.

\section{Sampling Techniques}

The researchers used Purposive sampling technique to select domain experts for knowledge acquisition and to collect previous tourist cases archived in Ethiopian ministry of culture and tourism. The selection criteria of domain experts for the study are based on the expertise, educational qualification level, year of experience and their immediate position. A total of six experts were selected from sector and they have been interviewed accordingly. These experts are consulted throughout the research work to evaluate the correctness of the acquired knowledge and to verify the cases acquired from the previous visitors cases.

\section{Knowledge (Case) Representation}

After the knowledge is acquired, the next task is knowledge (case) representation. Although there are various knowledge representation methods, like relational database knowledge representation, feature-value case representation, predicate based representation and soft computing knowledge representation methods have their own advantages and disadvantages. But for this research the researcher used feature-value case representation. The reason for representing the cases using feature-value representation is that this approach supports nearest neighbor retrieval algorithm and it represents cases in an easy way [17].This approach also uses old experiences to understand and solve new problems. It also reuses its solutions and lessons learned for future use. In addition, it represents cases in an easy way by using attribute and value pair representation [18]. The algorithms used to calculate the similarity of cases in a case base representation for this research are nearest neighbor retrieval algorithm. The similarity function of nearest neighbor retrieval algorithm involves in computing the similarity between the stored cases in the case base and the new query. After that, it selects the most similar stored cases to the query.

In the process of case based recommender system development, Knowledge representation is one of the basic steps. It refers to the formalism, both syntax and semantics, used to store knowledge in the architecture. It is also the process of interpreting domain knowledge into computer understandable form using various knowledge representation techniques. The object of a knowledge representation is to express knowledge in a computer tractable form, so that it can be used to enable our AI agents to perform well [18]. The common Knowledge representation techniques include semantic network, logics, rules, case base and frames. Among these, the 
researchers use case based representation method for this research.

\section{E. Development Tools}

To develop a recommender system there are various programming tools which are available both freely and commercially. Among this SWI-prolog, myCBR, and jCOLIBRI are the most widely used and known frameworks for teaching and academic research purpose. All of the aforementioned tools have their own capabilities and limitations.

According to [20], jCOLIBRI framework has the following features. Therefore for this research the researcher used jCOLIBRI framework due to the following unique capabilities of the tool.

- jCOLIBRI supports the full CBR cycles (Retrieval, Reuse, Revise and Retain).

- jCOLIBRI is extensible, reusable, different types of users and different purposes (development, research and/or teaching), compatible with commercial applications and, supporting different types of CBR systems, since it is just a .jar file suitable for web applications.

- It is suitable for developing large scale applications.

- jCOLIBRI works well in external database.
Once the prototype is developed, the functionality and user acceptance of the system should be tested. The evaluation processes focus on system's user acceptance of the prototype and the performance of the system. User acceptance measurements are concerned with issues how well the system addresses the needs of the user, whereas performance measurement determine if the system perform the required task successfully. In addition to this, the standard measures of relevance (performance of the system) in the information retrieval (precision, and recall) have been used to evaluate the performance of the prototype.

The researcher tested user acceptance of the system by involving evaluators using visual interaction methods together with questionnaire.

System evaluators were interacting with it by using appropriate cases. That is, sample cases has been selected purposely and then evaluators from the domain area were interacting with the system by taking a sample of test cases then, an experiment was conducted to know how new cases are matched with the cases from the case base using case similarity measurement. Each case are selected purposively and used to test the performance of the prototype. Based on that, they evaluate the performance of the system by using close ended questions. Recall and precision value of the system have been calculated based on its retrieval results.

\section{F. Testing/Evaluation}

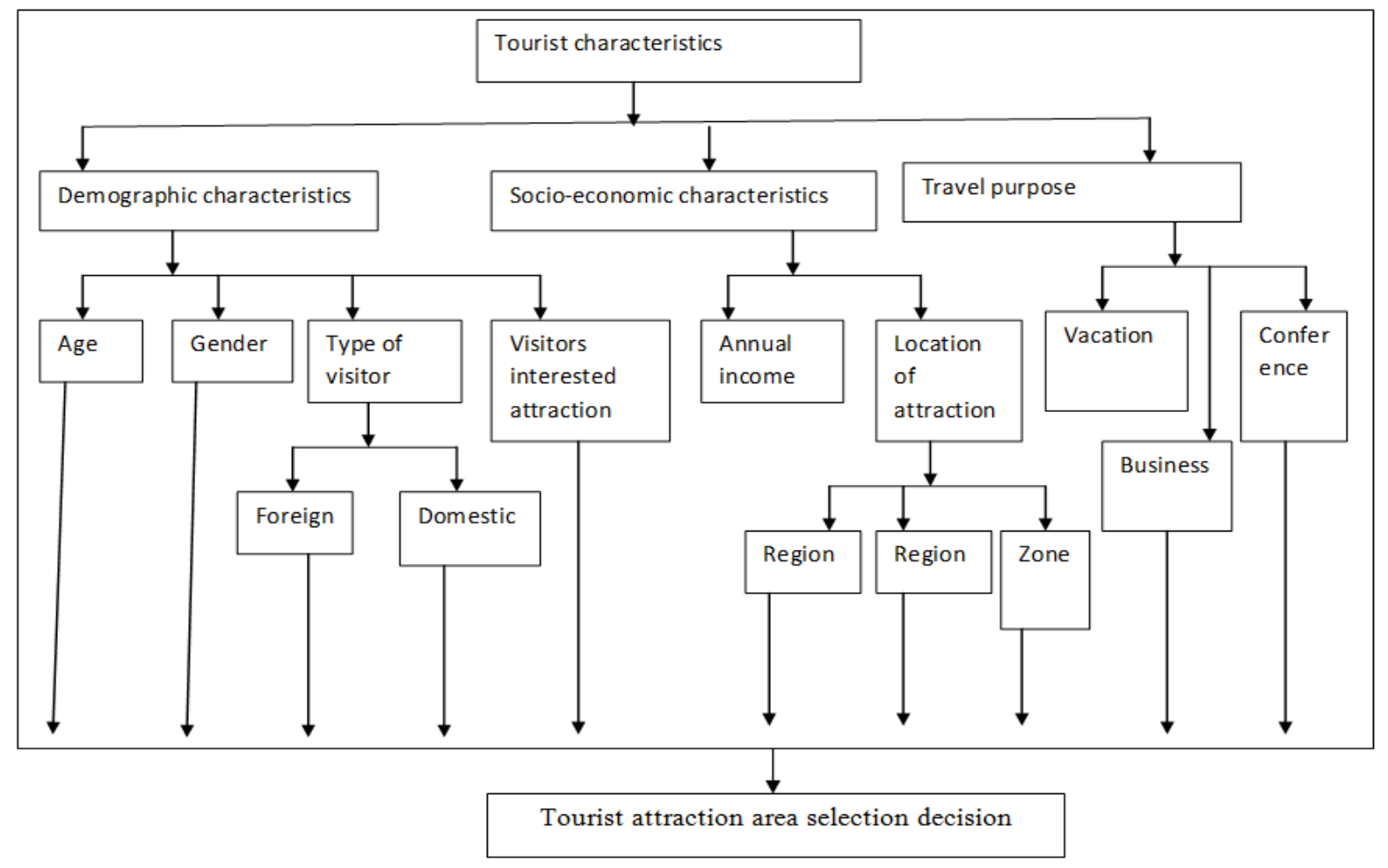

Fig.1. Hierarchical structure of tourist attraction area selection 


\section{PRototype DeVElopMent}

According to [21], Knowledge modeling is very significant for knowing the operational means in the development process of a knowledge-based system. It is also a vital stage of the knowledge engineering process. It can provide a means to easily understand the source of knowledge, the inputs and outputs of knowledge, and the designation of other parameters.

Although, there are various conceptual modeling techniques, for this study hierarchical tree structure is used to model how tourist attraction area selection is performed. The reason for using this one as modeling technique is, it easily model concepts and clearly explains the concepts in the problem area. It models the knowledge in the hierarchical manner. This model starts from the main concept at the highest level of the hierarchy and other sub concepts that can affect or affected by the highest level concept put next to down ward in the hierarchy.

\section{ARCHITECTURAL DESIGN OF CBR SYSTEM FOR TOURIST ATTRACTION AREA SELECTION}

As the new query (problem) is entered, the prototype of the system matches the new case with the solved case in the case base of the system by using similarity measurement. If relevant cases are found within the case base, then the prototype system ranks the relevant retrieved cases based on their local similarity. After that, the prototype by itself proposes a solution.

The proposed solution can be derived directly from a retrieved case that matches exactly or partially to the problem of the new case. Partially match of retrieved cases means some attribute values of the existing case and new cases (query) are the same and some attribute values are different. Using the proposed solutions directly may have a risk because some attribute values may need editing (changing) based on different conditions. As a result the user of the system should have made an adaptation on the proposed solution having differences between the proposed case and the new case. In addition to adaptation, case contradictions are revised. If there are situations where previous visitor's cases attribute values are not similar with the new case (query) attribute values. There is no similarity between the existing case and new case means there are no previous stored cases having similarity with the new case (query) in all attribute values. Therefore if there is no similarity between the existing and new case, the proposed solution cannot give recommendation to new cases. So during this time, this new case or problem of visitor can be revised and stored in the case base. Finally, the revised solution or stored cases is retained in the case base for problem solving in the next time.

Building of case based recommender system was started by collecting previously solved cases (i.e. previous visitor's cases) from NTO \&MoCT consisting of recorded data of visitors who are successful or satisfied in their recreational program. Since previously solved cases contains missing values and unnecessary information for this research, it need further processing in order to avoid such a problem and remove unnecessary attributes for tourist attraction area selection process. After processing of cases and selecting the most significant attributes, assigning weight and important parameters for each attribute was the next task which was performed.

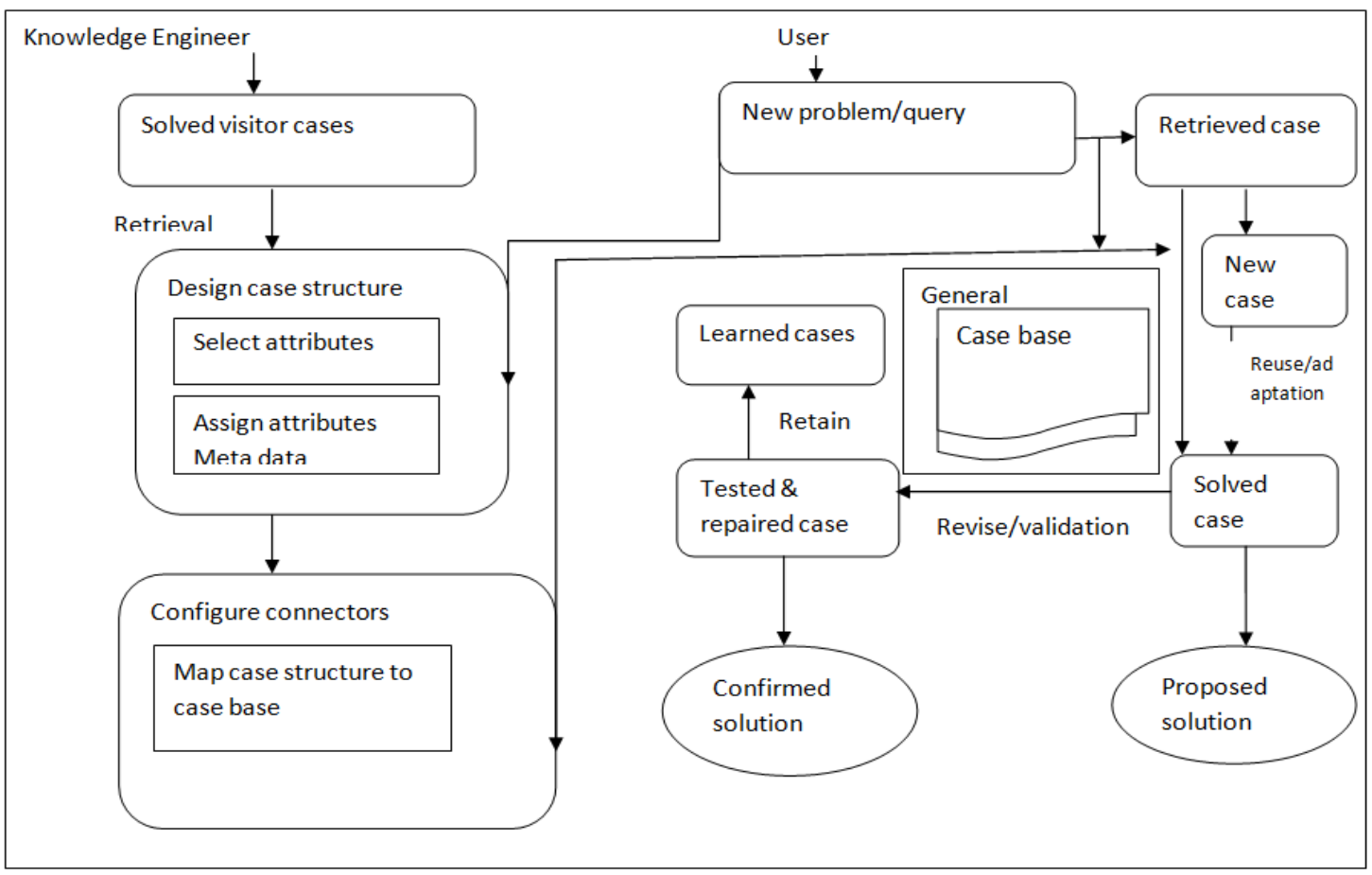

Fig.2. The proposed architecture of CBR in tourist attraction area selection process 
For the selection of important attributes that influence the recommendation of best tourist attraction area and visiting time, the researcher used data mining attribute selection algorithm called attribute selection algorithm. The reason for using attribute selection mechanism is since all attributes are not equally important to recommend tourist attraction areas and suitable visiting time to new visitors.

Once the case based recommender system is developed, users/tourists can use the system easily to choose their attraction areas based on the recommendation given by the system in order to retrieve the best cases that can match with their query. When users/visitors enter their query/case description through the user interface window, the system searches the best matching cases from the case base and retains the possible solution. If there is exact matching between the query and previous cases in the case base, the system recommends the most matched attraction area and visiting time for visitors. If the similarity between query and existing case is approximate, the proposed solution needs modification (adoption of solution) to fit the new case (query). At the end, the best modified solution should be stored into the case base for future use. The case base updates incrementally when the system learns from new case used by visitors.

\section{IMPLEMENTING THE CASE BASED RECOMMENDER SYSTEM}

After defining and configuring all the necessary steps required in designing case based recommender system using the programming tool JCOLIBRI, new case (query) entry application for new tourists is the next step as shown bellow.

As it is shown in the above query window (Fig. 4), visitors are expected to enter the query to each requested parameters or attributes in the space provided. After entering the query, at the bottom of the screen they will see the results of similar previous visitor cases and the recommended attraction areas, recommended visiting time, and explanation facility about the attraction areas on the execution log. For example in "Nationality" box visitors are required the query of their ethnic group as Ethiopian, Germany, Italy, Spain etc.

\section{EXPLANATION FACILITY}

One of the more interesting features of knowledge based systems is their ability to explain themselves. The explanation facility in this study is used to give explanation about the recommended attraction area after decision or recommendation is made by the system. Once the system reaches its final decision on the recommendation of attraction area and appropriate visiting time, the user may not have brief information about the recommended attraction area. In this case the system provides explanation facility about the recommended attraction area. Then the system gives more descriptions about the attraction areas such as the definitions, location, type of accommodations while visiting etc.

\section{Testing \& Evaluation}

Evaluation is an important issue for every system. The purpose of the evaluation process is to get the end user's views on the significance or usefulness of the system. The evaluation and testing issue of the system answers the question "To what extent the recommender system gives acceptable and accurate recommendation and explanation facility service to tourists/visitors?" To answer this question, system performance testing and user acceptance testing methods are used.

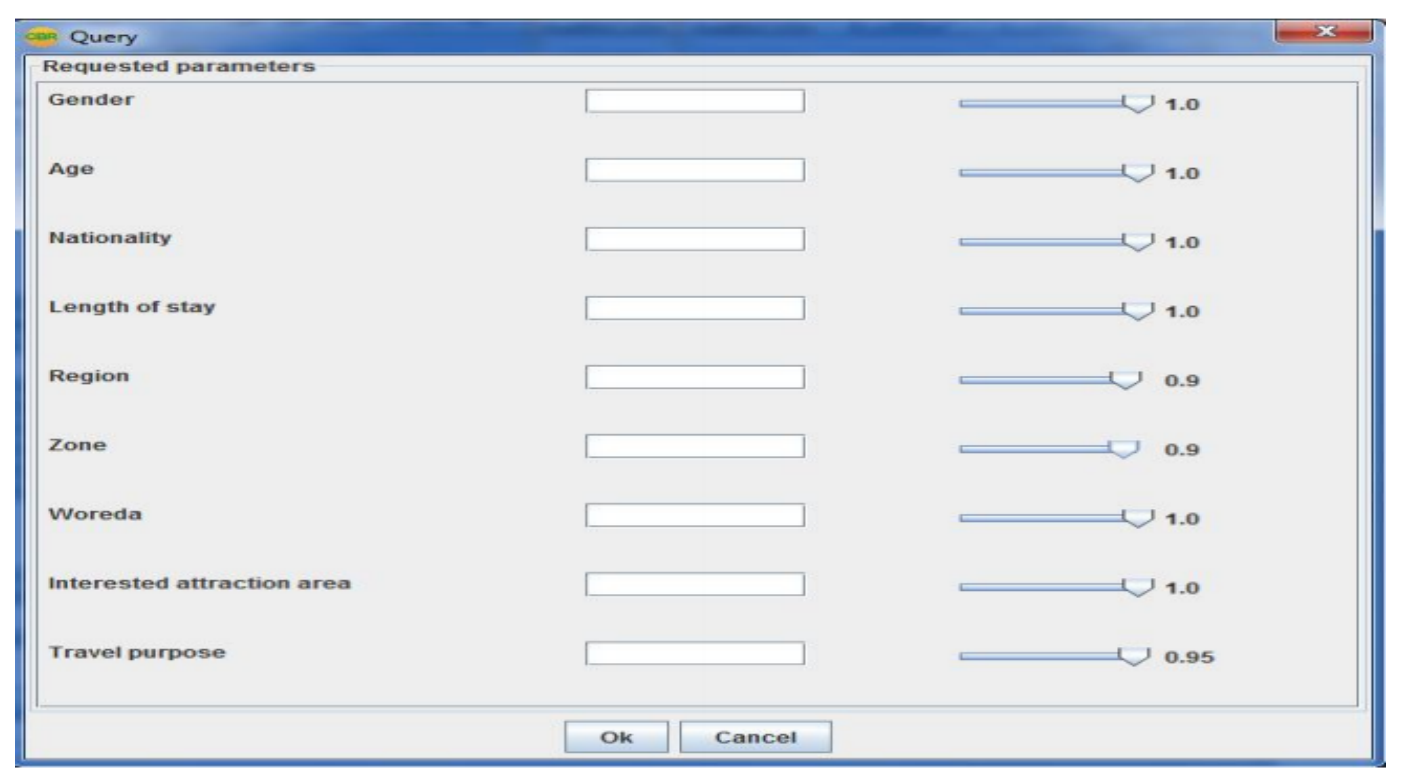

Fig.4. Windows for case entry in to the case base 


\section{A. Validating the Performance of CBR System}

The CBR engine has a built-in set of test cases in their case library. Effective use of this feature can facilitate the validation process by minimizing the involvement of domain experts in the process. Retrieval of previously stored cases to solve new problems is the first step in any CBR application. Retrieval of similar cases to the new case from previously solved cases is followed by the reuse of similar solutions. The CBR retrieval test is designed to evaluate the correctness of the retrieval function. To conduct the retrieval testing, for each test case the relevant visitors' cases from the case base should be identified. For identification of relevant cases, test cases are given to the domain expert in order to assign possible relevant cases from the case base to each of the test cases. The domain expert uses the recommendation value and solution attributes of the tourist cases as the main concept to assign the relevant case to the test cases. After the identification of the relevant cases to the test cases by the domain expert, precision and recall are calculated.

Table 1.Relevant Cases Assigned by Domain Experts for Sample Test Case.

\begin{tabular}{|c|c|}
\hline Test case & Relevant case from the case base \\
\hline Case 364 & $\begin{array}{l}\text { Case } 521 \text {, case } 19, \text { case } 273 \text {, case } 95 \text {, case } 476, \text { case } 314 \text {, case } 603 \text {, case } 66 \text {, case } 44\end{array}$ \\
\hline Case 277 & $\begin{array}{l}\text { Case } 29, \text { case } 550, \text { case } 423, \text { case } 615 \text {, case } 92, \\
\text { case } 478, \text { case } 73 \text {, case }\end{array}$ \\
\hline Case 472 & $\begin{array}{l}\text { Case } 381 \text {, case } 473 \text {, case } 88, \text { case } 576 \text {, case } 400, \\
\text { case } 562 \text {, case } 226 \text {, case } 12\end{array}$ \\
\hline Case 44 & $\begin{array}{l}\text { Case } 92, \text { case } 559, \text { case } 400, \text { case } 73 \text {, case } 562, \\
\text { case } 51 \text {, case } 231 \text {, case } 606 \text {, case } 17\end{array}$ \\
\hline Case 556 & $\begin{array}{l}\text { Case } 562 \text {, case } 73 \text {, case } 559 \text {, case } 400 \text {, case } 92 \text {, } \\
\text { case } 44 \text {, case } 605 \text {, case } 500\end{array}$ \\
\hline Case 600 & $\begin{array}{l}\text { Case } 17, \text { case, } 43 \text {, case } 604, \text { case } 605 \text {, case } 606, \\
\text { case } 20, \text { case } 99,\end{array}$ \\
\hline Case 12 & $\begin{array}{l}\text { Case } 226 \text {, case } 78, \text { case } 231 \text {, case } 233 \text {, case } 51 \text {, } \\
\text { case } 499, \text { case } 46, \text { case } 519\end{array}$ \\
\hline Case 311 & $\begin{array}{l}\text { Case } 51 \text {, case } 314 \text {, case } 312 \text {, case } 78 \text {, case } 66 \text {, case } \\
497 \text {, case } 19, \text {, case } 364 \text {, case } 13 \text {, case } 607 \text {, case } 2\end{array}$ \\
\hline
\end{tabular}

Once the relevant cases are identified and assigned to the test cases the next step is calculating the recall and precision value of the retrieval performance of the CBR system with a threshold interval. As indicated in the research of [22], there is no standard threshold for the degree of similarity that has been used for retrieving relevant cases in CBR. Different CBR researchers use different case similarity threshold. For this study, the threshold level of $[1.0,0.8)$ is adopted. This means cases with global similarity score greater than $80 \%$ are retrieved.

As shown in table above, the average recall and precision results were registered as $82 \%$ and $61 \%$ respectively which is a promising result and indicates that retrieval was done properly. For every test case more than average is registered for both recall and precision. But, precision is lower compared to the average recall. This is because of the tradeoff between precision and recall and small number of cases.
Table 2. Performance Measurement of the system using Precision and Recall

\begin{tabular}{|l|l|l|}
\hline Test case & Recall & Precision \\
\hline Case 364 & 0.7 & 0.71 \\
\hline Case 277 & 1.0 & 0.5 \\
\hline Case 472 & 0.87 & 0.57 \\
\hline Case 44 & 0.77 & 0.64 \\
\hline Case 556 & 0.87 & 0.57 \\
\hline Case 600 & 1.0 & 0.5 \\
\hline Case 12 & 0.87 & 0.57 \\
\hline Case 311 & 0.63 & 0.78 \\
\hline Average & 0.82 & 0.61 \\
\hline
\end{tabular}

\section{B. User acceptance testing}

User acceptance testing is the process of ensuring that whether the system satisfies the requirements of its endusers or not. This performed in real circumstances at National Tour Operation specifically with Tourism Experts and visitors. During testing the user acceptance, the applicability of the prototype is evaluated by potential users of the system. Potential users of the system are tourism experts and visitors. Case based reasoning system user acceptance evaluation method allows users (domain expert and visitors) to directly interact with the system and evaluate the performance of the case based system from the users' point of view. User acceptance testing helps to ensure the performance of the prototype by assessing the feedback acquired from the domain expert and visitors towards the developed prototype system. In order to evaluate the user acceptance of the developed prototype system, the researcher used questionnaire adapted from Ethiopia, (2002). To achieve the ambition of user acceptance evaluation of the prototype system, fourteen visitors and eight domain experts from NTO who were participating in different tourism sectors in the country were purposely selected For the ease of analyzing the performance of the system based on user's feedback, the researcher assigned numeric values to the five options as follows: Excellent=5, V. $\operatorname{good}=4$, Good=3, fair=2 and poor $=1$ for each of the criteria's.

Table 3. Evaluation Questions for user acceptance testing

\begin{tabular}{|l|l|}
\hline Question No & Evaluation Criteria \\
\hline Question1 & Easy to use of the recommender system \\
\hline Question2 & Is the system efficient in time system \\
\hline Question3 & Is the user interface interactive \\
\hline Question4 & Adequacy and clarity of the system \\
\hline Question5 & Relevancy of the retrieved case in the decision \\
\hline Question6 & $\begin{array}{l}\text { Fitness of the final solution to the new case } \\
\text { understand for new users }\end{array}$ \\
\hline Question7 & $\begin{array}{l}\text { Does the explanation facility give brief } \\
\text { description about the recommended attraction }\end{array}$ \\
\hline Question8 & $\begin{array}{l}\text { Rate the significance of the system in the } \\
\text { domain area }\end{array}$ \\
\hline Question 9 & $\begin{array}{l}\text { Relevancy of attributes in representing } \\
\text { visitors case }\end{array}$ \\
\hline
\end{tabular}


Table 4. Performance evaluation by visitor.

\begin{tabular}{|l|l|l|l|l|l|l|l|}
\hline $\begin{array}{l}\text { Quer } \\
\text { y }\end{array}$ & $\begin{array}{l}\text { Excel } \\
\text { lent }\end{array}$ & V.good & Good & $\begin{array}{l}\text { Fai } \\
\text { r }\end{array}$ & $\begin{array}{l}\text { Po } \\
\text { or }\end{array}$ & $\begin{array}{l}\text { Ave } \\
\text { rage }\end{array}$ & $\%$ \\
\hline 1 & 2 & 9 & 3 & - & - & 3.9 & 78 \\
\hline 2 & 6 & 8 & - & - & - & 4.4 & 84 \\
\hline 3 & 2 & 12 & - & - & - & 4.1 & 82 \\
\hline 4 & 3 & 7 & 4 & - & - & 3.9 & 78 \\
\hline 5 & 3 & 9 & 2 & - & - & 4.0 & 80 \\
\hline 6 & 4 & 7 & 3 & - & - & 4.0 & 80 \\
\hline 7 & 1 & 8 & 5 & - & - & 3.7 & 74 \\
\hline 8 & 1 & 13 & - & - & - & 4.9 & 98 \\
\hline & 3 & 11 & - & - & - & 4.2 & 84 \\
\hline &
\end{tabular}

The average value of each question is calculated using the sum of values of Excellent, V.good, good, fair and poor.

As it is shown in the above table, $21.4 \%$ of the respondents rated the easiness of the recommender system as good, $64.2 \%$ of them rate as very good and the remaining $14.2 \%$ rated as excellent. In case of efficiency of the system in terms of time, $57.14 \%$ of the respondents were rated as very good, and the remaining $42.85 \%$ of them rated as excellent. Regarding to the interactivity of the prototype, $85.71 \%$ of the respondents rated as very good and the remaining $14.28 \%$ of them rated as excellent. In the case of adequacy and clarity of the system, $28.57 \%$ of the respondents rated as good, $50 \%$ of them rated as very good and the remaining $21.42 \%$ of the respondents rated as excellent .In the same way, $92.85 \%$ of the respondents rate the applicability of the prototype in their domain area as excellent and the remaining $7.14 \%$ of the respondent's rate as very good. Generally, it was found that most evaluators have positive feedback about recommender system. The average performance of the system according to the evaluation results filled by the domain experts is 4.2 out of 5 or $82 \%$ which shows a promising result and users are satisfied with the recommender system.

\section{RESUltS AND DisCUSSIONS}

In this study, both system performance testing and user acceptance testing have been done for the prototype recommender system. In measuring the performance (accuracy) of the system, the CBR modules are validated. The accuracy of CBR module is calculated as $85.4 \%$. In addition to accuracy, user acceptance evaluation of the prototype system has been calculated as $82 \%$. The researchers faced some challenges during the study which limits the recommender system to register a better performance for recommending tourist attraction area. These are discussed as follows: The tacit knowledge elicited from the domain experts, the explicit knowledge acquired from documented sources and cases collected from the different tourist cases were in the form of paper printed format. During the system development process, it was difficult for the researcher to convert all the needed knowledge into the electronically recorded format because it needs a lot of data preprocessing and this task was also tough. The performance of the prototype system depends directly on the quality of the knowledge acquired from domain experts. However, knowledge elicitation from domain experts was the most difficult task due to the fact that, tacit knowledge is difficult to transfer to another person.

\section{CONCLUSION AND FUTURE WORK}

Recommender systems have proven to be an important response to the problem of information overload by providing users with more proactive and personalized information services. CBR enables people to make decision from the past solved cases i.e. a new problem is solved by finding a similar past case and reusing it in the new problem circumstances. CBR can work with new cases that match partially to the case from the case base as compared to rule based reasoning. Though, rule based reasoning cannot solve a problem that doesn't exactly match with the rule of the system. This shows that rule based reasoning works in a closed assumption where every fact are known and represented.

As studies shown that, the advising services given on the area of tourism in Ethiopia is in its infant stage. There are various factors that affect the tourism sector to be in its infant stage. Among these, shortage of skilled man power in the area, lack of guide line or criteria to assign visitors in different attraction area, absence of experts that can provides consistency advising service, and lack awareness on the side of visitors about the purpose of advising systems for the selection of tourist attraction area and visiting time.

In this paper a recommender system that can provide possible recommendation system for visitors has been developed. The system is evaluated using different evaluation methods and achieved $85.4 \%$ of an average performance.

The relevant attributes used for this research were collected from the previous tourist cases from NTO and MoCT. These attributes are not sufficient for the selection of attraction area and visiting time decision. So, further research can be done by adding other important attributes such as housing preference, level of education, marital status, and purchasing habits by making a direct survey of successful visitors.

\section{REFERENCES}

[1] Angela Edmunds, Anne Morris (2000). The problem of information overload in business organizations: Department of Information Science, Loughborough University.

[2] Burke, R. (2007). Hybrid Web Recommender Systems, University of California, Irvine.

[3] Culture and tourism office (2011). Tourism Development Strategy, Ethiopia, Addis Ababa.

[4] Ethiopia T., (2002). Application of Case-based Reasoning for Amharic Legal Precedent Retrieval: A Case Study with the Ethiopian Labor Law, Addis Ababa University, Ethiopia.

[5] Fabiana, et al (2003). Case-based recommender systems: A unifying view, in Intelligent Techniques for Web Personalization, IJCAI 2003 Workshop, p. 2-10. 
[6] YechaleMehiret (2011). Tourism certification as a tool for promoting sustainability in the Ethiopian tourism industry. Addis Ababa University, Addis Ababa, Ethiopia.

[7] [Ministry of culture and tourism (2012). Manuals of Ethiopian tourism guide, Ethiopia, Addis Ababa.

[8] Main, et al (2001). A tutorial on case based reasoning, pp.1-28.

[9] Shimazu (2002). A Conversational Case-based Reasoning Tool for Developing Salesclerk Agents in E-Commerce Webshops. Artificial Intelligence Review 18 (3-4), 223244.

[10] Ralph Bergmann (1998). Introduction to Case-Based Reasoning, Department of Computer Science University of Kaiserslautern.

[11] Prentza andHatzilygeroudi (2007). Categorizing approaches combining rule-based and case-based reasoning, Department of Computer Engineering and Informatics, School of Engineering, University of Patras.

[12] United Nations World Tourism Commission (2007). Tourism Highlights. 2007 Edition.

[13] Aamodt, A., Plaza, E. (1994). Case-Based Reasoning: Foundational Issues, Methodological

[14] Variations and System Approaches. AI Communications. IOS Press, Vol. 7: 1, pp. 39-59.

[15] Fong, S. and Biuk-Aghai, R. (2009). An Automated Admission Recommender system for Secondary School Student.The 6th international conference on Information Technology and application.

[16] Satyanarayana, K. and Rajagoplan, S.P. (2007). Recommender system for Educational Institutions.Asian Journal of Information Technology. M.G.R University, India.

[17] Bendakir, N. and 1meur, E. (2006). Using Association Rules for Course Recommendation.American Association for Artificial Intelligence.

[18] Salem, et al (2005). A Case Base Experts System for Diagnosis of Heart Disease. International Journal on Artificial Intelligence and Machine Learning, 5(1), pp. 33-39.

[19] Bergmann, et al (2005). a representation in case based reasoning, The Knowledge Engineering Review, Vol. $00: 0,1-4$.

[20] Burke, R. (2006). Knowledge based recommender systems, University of California, Irvine.

[21] Juan A., et al (2002). jCOLIBRI 1.0 in a nutshell. A software tool for designing CBR systems.
[22] Tehrani, et al (2009). A Conceptual Model of Knowledge Elicitation, College of Business, Technology and Communication, pp. 2.

[23] Getachew W. (2012).Application of case-based reasoning for anxiety disorder diagnosis, Addis Ababa University, Ethiopia.

\section{Author's profile}

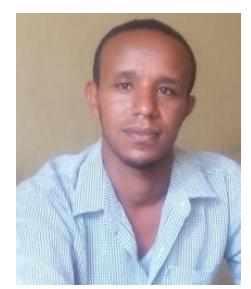

Mr. Tamir Anteneh obtained his M.S.c degree in information Science from Addis ababa University and B.S.c degree in Information Technology from Bahir Dar University, Ethiopia. $\mathrm{He}$ is currently working as a lecturer at the faculty of computing, Bahir Dar University. His area of research interests include Information retrieval, machine leaning, cloud computing, Databases and knowledge based systems.

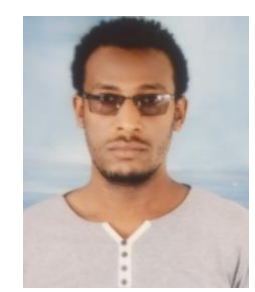

Mr. Alemu Kumilachew obtained his M.S.c degree in Information Science from Addis Ababa University and B.S.c degree in Information Technology from Jimma University, Ethiopia. $\mathrm{He}$ is currently working as a lecturer at the faculty of computing, Bahir Dar University. His area of research interests include information systems and retrieval, Artificial intelligence, question answering systems, machine learning

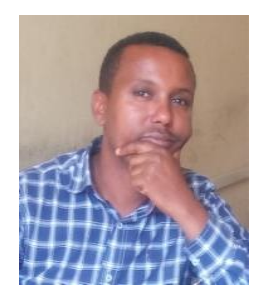

Mr. Adane Nega obtained his M.S.c degree in information technology from university of Gondar and B.S.c degree in computer science from Bahir Dar University, Ethiopia. $\mathrm{He}$ is currently working as a lecturer at the faculty of computing, Bahir Dar University. His area of research interests include Artificial intelligence and soft computing, Data mining, big data analysis, machine learning.

How to cite this paper: Tamir Anteneh Alemu, Alemu Kumilachew Tegegne, Adane Nega Tarekegn, "Recommender System in Tourism Using Case based Reasoning Approach", International Journal of Information Engineering and Electronic Business(IJIEEB), Vol.9, No.5, pp. 34-43, 2017. DOI: 10.5815/ijieeb.2017.05.05 